\title{
Esquema de Diversidade Cooperativa com Conhecimento Parcial do Canal nos Nós Cooperadores
}

\author{
Renato Machado e Bartolomeu F. Uchôa Filho
}

\begin{abstract}
Resumo-Neste artigo, propõe-se um esquema simples de diversidade cooperativa para um sistema de comunicação que consiste em dois nós cooperadores, que recebem um único bit de realimentação do nó destino com informação sobre o estado do canal. Essencialmente, o bit de realimentação informa qual é o nó cooperador que apresenta o canal mais forte e esta informação é usada apropriadamente para se obter diversidade cooperativa. Um receptor linear é proposto e seu desempenho de erro se mostra ser muito próximo ao do detector de máxima verossimilhança. Deriva-se um limitante superior da probabilidade de erro média para a modulação BPSK e canal com desvanecimento Rayleigh plano, assumindo-se condições ideais para os canais inter-usuários.
\end{abstract}

Palavras-Chave-Canal de realimentação limitado, códigos espácio-temporais, diversidade cooperativa, seleção de nós.

Abstract-In this paper, we propose a simple cooperative diversity scheme for a communication system consisting of two cooperating nodes that receive a single channel state information (CSI) bit from the destination node. Essentially, the feedback bit tells which cooperating node has the strongest channel, and this information is used appropriately to obtain cooperative diversity. A simple linear receiver is proposed and its performance is shown to be very close to the maximum-likelihood performance. An upper bound on the average error probability is derived for binary phase-shift keying (BPSK) in flat Rayleigh channels under the assumption of ideal inter-user channel.

Keywords - Cooperative diversity, node selection, limited feedback, space-time code.

\section{InTRODUÇÃo}

Já é bem conhecido que sistemas de comunicação sem fio com múltiplas antenas transmissoras e múltiplas antenas receptoras (MIMO) (multiple-input multiple-output) possuem capacidade de canal elevada [1], [2]. Técnicas com múltiplas antenas são muito atrativas por serem empregadas em estações rádio-base com aplicações para celulares, as quais já foram incluídas nos padrões de comunicação sem fio de terceira geração. Infelizmente, em muitos cenários de comunicação sem fio, os transmissores são muito pequenos e não possibilitam a instalação de múltiplas antenas. Para superar essa limitação, esquemas com diversidade cooperativa vêm sendo propostos [3], [4].

A idéia básica por trás de comunicação cooperativa reside na observação que, em um ambiente de comunicação sem fio, o sinal transmitido pelos nós fontes são ocasionalmente

R. Machado e B. F. Uchôa-Filho estão com o Grupo de Pesquisa em Comunicações, Departamento de Engenharia Elétrica, Universidade Federal de Santa Catarina, Florianópolis, SC, 88040-900, BRASIL. e-mail: \{machado, uchoa\}@eel.ufsc.br "ouvidos" por outros nós, os quais podem ser vistos como "parceiros". A fonte e seus parceiros podem conjuntamente processar e transmitir suas informações criando assim uma "rede virtual de antenas", embora cada um desses nós estejam equipados com apenas uma única antena. É o chamado caso MIMO distribuído.

Desde o trabalho de Sendonaris et al. [3], [4], o interesse em comunicação cooperativa começou a crescer. Sendonaris et al. propuseram algoritmos interessantes de cooperação para sistemas de transmissão to tipo CDMA (code-division multipleaccess), onde cada unidade móvel decodifica e repassa um certo número de bits recebidos de seu parceiro. Também contribuindo para o aumento do interesse nessa área, Laneman et al. [5] apresentaram o primeiro trabalho em comunicação cooperativa considerando certos aspectos da teoria da informação, examinando regiões de taxas de transmissão alcançáveis e probabilidades outages. Em [5], mostrou-se que tanto a técnica amplifica e encaminha (amplify-and-forward) quanto métodos adaptativos alcançam ordem de diversidade igual a dois para sistemas de cooperação com dois usuários. Laneman et al. [6] também sugeriram o emprego de códigos espácio-temporais de bloco "convencionais" (originalmente proposto em [7], [8]) em um cenário "distribuído" de cooperação.

Paralelamente a [6], uma abordagem alternativa chamada de cooperação codificada foi proposta em [9], em que a transmissão cooperativa é integrada à codificação de canal. Em [9], a idéia é que cada usuário, ao invés de repetir os bits recebidos (ou via amplificação, ou via decodificação), tente enviar alguma redundância adicional para o(s) seu(s) parceiro(s). A maioria dos trabalhos em que os nós cooperadores atuam tanto como fontes de dados quanto como relays é para esquemas de transmissão to tipo CDMA ou TDMA (time-division multipleaccess).

Um sistema de comunicação sem fio pode obter significantes melhorias em desempenho quando o conhecimento do canal é disponibilizado nos transmissores [10], [11]. Em [12], [13], um canal de realimentação é usado para se explorar cooperação. Ahmed et al. [14] consideraram métodos práticos para aproximar os limites de desempenho teóricos em um canal relay com desvanecimento, sob diferentes considerações sobre o conhecimento do estado do canal no transmissor, para um cenário típico com canal relay (i.e., fonte-relaydestino). Objetivando-se explorar plenamente os benefícios de uma transmissão cooperativa, deve-se explorar o canal de realimentação sempre que este estiver disponível.

Neste artigo, propõe-se um esquema de diversidade coope- 


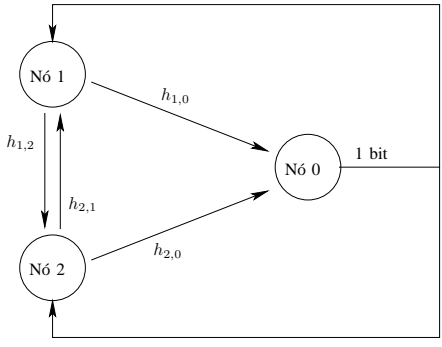

Fig. 1. Diagrama de blocos do esquema de cooperação proposto.

rativa para $N_{T}=2$ nós transmissores e $N_{D}=1$ nó destino, todos equipados com uma única antena. Antes de começar qualquer transmissão de dados, os nós transmissores recebem 1 bit de realimentação, que traz informação sobre o estado do canal. Vale ressaltar que a mesma informação sobre o estado do canal é passada para os dois nós cooperadores. Esse fato, aliado ao fato de que o canal permanece constante por um certo intervalo de tempo, resulta em um overhead desprezível na prática.

Os canais entre os nós transmissores (canais inter-usuários) e entre cada nó transmissor e o nó destino (canais diretos) são considerados mutuamente independentes. $\mathrm{O}$ valor do bit de realimentação indica qual dos canais diretos é o melhor. Porém, não é possível se aplicar diretamente a técnica de seleção combinada e simplesmente transmitir ambos símbolos pelo mesmo nó fonte, uma vez que cada símbolo só está disponível para o seu respectivo nó antes da transmissão começar. O nó associado ao pior canal deve utilizar o mínimo de potência possível, o suficiente para assegurar que o símbolo transmitido por ele será ouvido pelo nó cooperador.

Na próxima seção, descreve-se o modelo do sistema. O novo esquema de diversidade cooperativa é descrito na Seção III. $\mathrm{Na}$ Seção IV, apresenta-se uma análise da probabilidade de erro para o esquema proposto, assumindo-se uma condição ideal para o canal inter-usuário. $\mathrm{Na}$ Seção $\mathrm{V}$, resultados de simulação são apresentados. Finalmente, na Seção VI, consideram-se algumas conclusões e comentários finais.

\section{Modelo do Sistema}

O sistema de comunicação sem fio com cooperação considerado neste artigo é mostrado na Figura 1.

Ele consiste em $N_{T}=2$ nós transmissores (nós parceiros) e $N_{D}=1$ nó receptor (nó destino), todos equipados com uma única antena. Nesta seção, e nas Seções III e IV, por simplicidade, considera-se que os nós parceiros estão próximos entre si, podendo-se, consequientemente, assumir que o canal de comunicação entre o nó transmissor e seu parceiro é confiável. Assume-se que os canais diretos sofrem desvanecimento Rayleigh e plano. Os sinais recebidos pelo nó receptor são contaminados por um ruído aditivo Gaussiano branco (AWGN) (additive white Gaussian noise). Os nós transmissores, denominados nó 1 e nó 2 , enviam seus símbolos de informação para o nó destino, denominado nó 0. Os nós transmissores também se comunicam entre si. Os coeficientes do desvanecimentos $h_{i, 0}$ associados aos canais entre os nós $i$ e o nó $0, i=1,2$, são modelados como variáveis aleatórias
Gaussianas com média zero e variância $1 / 2$ por dimensão complexa. Assume-se que os coeficientes dos desvanecimentos são constantes durante a transmissão de um bloco de dois símbolos consecutivos, mudando aleatóriamente a cada novo bloco de transmissão.

Assume-se também que os coeficientes dos desvanecimentos dos canais diretos são conhecidos pelo receptor e que um canal de realimentação, totalmente livre de erros e de atrasos, através do qual $b$ bits possam ser enviados para os nós transmissores. Além disso, assume-se que a informação trazida pelo canal de realimentação chegue aos nós transmissores antes mesmo que a primeira transmissão seja realizada. Em outras palavras, os $b$ bits de realimentação são usados para selecionar os modos de transmissão para ambos períodos de símbolo dentro de um bloco de transmissão.

\section{Esquema De Diversidade Cooperativa Proposto COM UM BIT DE REALIMENTAÇÃO}

Nesta seção, o esquema de diversidade cooperativa proposto é apresentado para um sistema de comunicação cujo modelo foi descrito na seção anterior, para $b=1$ bit de realimentação. A transmissão dentro de um bloco é realizada da seguinte maneira. No primeiro período de símbolo, cada nó transmissor envia seu próprio símbolo de informação para o nó destino, e este símbolo é recebido pelo nó parceiro também, que realiza sua detecção. No segundo período de símbolo, os nós transmissores realizam cooperação e retransmitem a informação recebida de seu nó parceiro conforme é descrito abaixo.

No esquema proposto, uma alocação de potência não uniforme é empregada nos nós transmissores. Denotam-se $P_{+} \mathrm{e}$ $P_{-}\left(P_{+}>P_{-}\right)$as potências de transmissão alocadas nos nós transmissores, sendo que a restrição de potência $P_{+}+P_{-}=P$ é imposta, em que $P$ é a potência total transmitida. $O$ valor da potência alocada em cada nó transmissor poderia variar a cada bloco de transmissão, dependendo das condições instantâneas do canal. No entanto, isto requereria um número maior de bits de realimentação. Ao invés disso, considera-se uma alocação com dois níveis de potência, a saber, $\alpha$ e $P-\alpha$, em que $\alpha$ é um valor otimizado à parte e permanece fixo por todo o tempo. Os dois níveis (complementares) de potência são designados alternadamente para os nós 1 e 2 , de acordo com o valor do bit de realimentação recebido. Em função de se ter considerado o canal inter-usuário totalmente sem ruído (ideal), qualquer quantidade de potência positiva $P_{-}$torna-se suficiente para se detectar corretamente o símbolo vindo do nó parceiro. Assume-se para o restante desse artigo que $P_{+} \gg P_{-}$. Vale a pena acrescentar que esta última solicitação é bem razoável, considerando-se que os nós transmissores estão próximos um dos outros, mesmo quando os canais inter-usuários não sejam ideais.

\section{A. O transmissor}

Sejam $s_{1}$ e $s_{2}$ os símbolos de dados gerados pelos nós 1 e 2, respectivamente, ambos pertencentes a uma constelação de símbolos com energia média unitária. Primeiro, suponha que $\left|h_{1,0}\right|>\left|h_{2,0}\right|$, em que $|\cdot|$ denota o valor absoluto. Nesse caso, uma maior potência deve ser alocada ao nó 1, e 
TABELA I

ESQUEMA DE TRANSMISSÃO

\begin{tabular}{||c|c|c|c|c|c|c||}
\hline \multicolumn{3}{|c|}{ Se $\left|h_{1,0}\right|>\left|h_{2,0}\right|$} & \multicolumn{3}{|c||}{ Se $\left|h_{2,0}\right|>\left|h_{1,0}\right|$} \\
\hline \hline Nó & Instante 1 & Instante 2 & Nó & Instante 1 & Instante 2 \\
\hline \hline 1 & $\sqrt{\overline{P_{+}}} s_{1}$ & $\sqrt{P} s_{2}$ & 1 & $\sqrt{\overline{P_{-}}} s_{1}$ & 0 \\
\hline 2 & $\sqrt{\overline{P_{-}}} s_{2}$ & 0 & 2 & $\sqrt{P_{+}} s_{2}$ & $\sqrt{P} s_{1}$ \\
\hline \hline
\end{tabular}

a transmissão em nosso esquema de diversidade cooperativa é descrito na Tabela I. Note que, no segundo período de símbolo, somente o nó cooperador 1 realiza a transmissão, com potência total. Na prática, este símbolo transmitido pelo nó 1 é a decisão tomada em relação ao símbolo que foi transmitido pelo nó 2 no primeiro período de símbolo. Uma vez que está sendo considerado que o canal inter-usuário é totalmente livre de erros, pode-se assumir que o símbolo $s_{2}$ é transmitido pelo nó 1 no período de símbolo cooperativo.

Quando $\left|h_{2,0}\right|>\left|h_{1,0}\right|$, a maior porcentagem da potência de transmissão deve ser alocada ao nó 2 , e a transmissão ocorre conforme apresentado na Tabela I. Devido à simetria da Tabela I, assume-se para o restante desta seção que $\left|h_{1,0}\right|>\left|h_{2,0}\right|$.

\section{B. Recepção de Máxima Verossimilhança}

Os símbolos recebidos no nó receptor nos instantes 1 e 2 são dados por

$$
\begin{aligned}
& y_{1}=\sqrt{P_{+}} s_{1} h_{1,0}+\sqrt{P_{-}} s_{2} h_{2,0}+\eta_{1} \\
& y_{2}=\sqrt{P} s_{2} h_{1,0}+\eta_{2},
\end{aligned}
$$

respectivamente, em que $\eta_{1}$ e $\eta_{2}$ representam o ruído AWGN, modelados como variáveis aleatórias complexas com média zero e variância $N_{0} / 2$ por dimensão, para os instantes 1 e 2 , respectivamente.

A decisão de máxima-verossimilhança (ML) $\left(\tilde{s}_{1}, \tilde{s}_{2}\right)$ é dada por

$$
\begin{gathered}
\left(\tilde{s}_{1}, \tilde{s}_{2}\right)=\arg \min _{s_{1}, s_{2}}\left|y_{1}-\sqrt{P_{+}} s_{1} h_{1,0}-\sqrt{P_{-}} s_{2} h_{2,0}\right|^{2} \\
+\left|y_{2}-\sqrt{P} s_{2} h_{1,0}\right|^{2} .
\end{gathered}
$$

Assumindo-se que $P_{+} \gg P_{-}$, o receptor de ML pode ser simplificado. Expandindo-se o termo

$$
\left|y_{1}-\sqrt{P_{+}} s_{1} h_{1,0}-\sqrt{P_{-}} s_{2} h_{2,0}\right|^{2}
$$

de (2), obtém-se

$$
\begin{aligned}
& \left|y_{1}\right|^{2}+P_{+}\left|s_{1}\right|^{2}\left|h_{1,0}\right|^{2}+P_{-}\left|s_{2}\right|^{2}\left|h_{2,0}\right|^{2} \\
& +\sqrt{P_{+}} \sqrt{P_{-}}\left(s_{1} s_{2}^{*} h_{1,0} h_{2,0}^{*}+s_{2} s_{1}^{*} h_{2,0} h_{1,0}^{*}\right) \\
& \quad-2 \sqrt{P_{+}} \Re\left\{y_{1} s_{1}^{*} h_{1,0}^{*}\right\}-2 \sqrt{P_{-}} \Re\left\{y_{1} s_{2}^{*} h_{2,0}^{*}\right\},
\end{aligned}
$$

em que $*$ denota conjugado complexo e $\mathfrak{R}\{\cdot\}$ entende-se por parte real. Sendo $P_{+} \gg P_{-}$, pode-se negligenciar os termos multiplicados por $P_{-}, \sqrt{P_{-}}$e $\sqrt{P_{+}} \sqrt{P_{-}}$, resultando na seguinte aproximação

$$
\begin{aligned}
& \left|y_{1}-\sqrt{P_{+}} s_{1} h_{1,0}-\sqrt{P_{-}} s_{2} h_{2,0}\right|^{2} \approx \\
& \quad\left|y_{1}\right|^{2}+P_{+}\left|s_{1}\right|^{2}\left|h_{1,0}\right|^{2}-2 \sqrt{P_{+}} \Re\left\{y_{1} s_{1}^{*} h_{1,0}^{*}\right\} .
\end{aligned}
$$

Nota-se que o primeiro termo de (2) é independente de $s_{2}$ quando $P_{+} \gg P_{-}$. Logo, pode-se concluir que realizar a recepção de ML dos símbolos $s_{1}$ e $s_{2}$ independentemente, é praticamente o mesmo que realizar a recepção de ML em (2). A partir desta observação é possível se propor um receptor alternativo que ofereça um desempenho muito próximo ao do receptor de ML.

\section{Receptor Alternativo de Baixa Complexidade}

$\mathrm{O}$ primeiro receptor alternativo aqui proposto realiza a detecção de ML de $s_{2}$ baseado na seguinte minimização

$$
\begin{aligned}
\hat{s}_{2} & =\arg \min _{s_{2}}\left|y_{2}-\sqrt{P} s_{2} h_{1,0}\right|^{2} \\
& =\arg \min _{s_{2}}\left|y_{2}\right|^{2}+P\left|s_{2}\right|^{2}\left|h_{1,0}\right|^{2}-2 \sqrt{P} \Re\left\{y_{2} s_{2}^{*} h_{1,0}^{*}\right\},
\end{aligned}
$$

a qual corresponde em minimizar o segundo termo em (2). Dessa maneira, $s_{1}$ poderia ser detectado removendo-se de $y_{1}$ a interferência causada por $s_{2}$. Para tal, o símbolo $\hat{s}_{2}$, detectado no passo anterior, poderia ser usado e a decisão em $s_{1}$ seria

$$
\hat{s}_{1}=\arg \min _{s_{1}}\left|y_{1}-\sqrt{P_{+}} s_{1} h_{1,0}-\sqrt{P_{-}} \hat{s_{2}} h_{2,0}\right|^{2} .
$$

No entanto, a partir de (3), a minimização em (5) pode ser aproximadamente obtida por

$$
\hat{s}_{1}=\arg \min _{s_{1}}\left|y_{1}\right|^{2}+P_{+}\left|s_{1}\right|^{2}\left|h_{1,0}\right|^{2}-2 \sqrt{P_{+}} \Re\left\{y_{1} s_{1}^{*} h_{1,0}^{*}\right\} \text {. }
$$

Como um resultado, (6) e (4) podem ser obtidas em paralelo.

Agora, pode-se fazer uma observação que nos permite concluir que o esquema proposto possui um desempenho de erro muito bom. Em particular, uma ordem de diversidade cooperativa igual a 2 é alcançada. Note que, desde que é assumido $P_{+} \gg P_{-}$e $\left|h_{1,0}\right|>\left|h_{2,0}\right|$, é possível se negligenciar o terceiro termo dentro do módulo quadrático em (5). Então, a desigualdade $\left|h_{1,0}\right|>\left|h_{2,0}\right|$ implica que (5) corresponde ao receptor de ML de um esquema de seleção em que o símbolo $s_{1}$ é transmitido com quase toda energia de transmissão. Semelhantemente, (4) corresponde à detecção de ML de um esquema de seleção em que o símbolo $s_{2}$ é transmitido com potência total $P$. Assim, a ordem de diversidade do esquema cooperativo proposto é semelhante à do esquema com diversidade de seleção de ordem 2.

Se ainda considerarmos que todos os sinais transmitidos pelos dois nós transmissores possuem a mesma energia, que é o caso se uma constelação PSK for adotada, então o receptor proposto torna-se linear e reduz-se a

$$
\begin{aligned}
& \hat{s}_{1}=\arg \max _{s_{1}} \mathfrak{R}\left\{y_{1} s_{1}^{*} h_{1,0}^{*}\right\} \\
& \hat{s}_{2}=\arg \max _{s_{2}} \mathfrak{R}\left\{y_{2} s_{2}^{*} h_{1,0}^{*}\right\} .
\end{aligned}
$$

Adota-se para o restante desse artigo a modulação BPSK (binary phase shift keying), permitindo-nos referenciar o receptor proposto como um detector linear (LD). O receptor de ML será referido aqui como MLD (maximum likelihood detector).

Na próxima seção, um limitante superior para a probabilidade de erro média do receptor alternativo descrito em (4) e (6) para a modulação BPSK é derivada. Os resultados obtidos através de simulações são apresentados na Seção V. 


\section{ANÁLISE De DESEMPENHo}

Assume-se que os canais inter-usuários são confiáveis, i.e., o símbolo transmitido pelo nó transmissor com o melhor canal no segundo período de símbolo é o mesmo símbolo transmitido pelo seu nó parceiro no primeiro período de símbolo. Assume-se, também, que a modulação BPSK é usada pelos nós transmissores. Define-se $h_{\max }$ (resp. $h_{\min }$ ) como o coeficiente do canal direto com maior (resp. menor) módulo quadrático instantâneo. Sob essas condições, deriva-se a taxa de erro de bit para o receptor linear apresentado na Seção III-C.

Inicia-se a análise calculando-se a expressão exata da taxa de erro de bit (BER) (bit error rate) média para a modulação BPSK de um esquema de diversidade de seleção de ordem 2 , que é dada por [19, eq. (9.213), com $\rho=0$ e $g=1$ ]:

$$
\begin{aligned}
P_{e}\left(\gamma_{0}\right) & =\int_{0}^{\infty} Q\left(\sqrt{2 \gamma_{b}}\right) p\left(\gamma_{b}\right) d \gamma_{b} \\
& =\left(1-\sqrt{\frac{\gamma_{0}}{\gamma_{0}+1}}\right)-\frac{1}{2}\left(1-\sqrt{\frac{\gamma_{0}}{\gamma_{0}+2}}\right)
\end{aligned}
$$

em que $\gamma_{b}=\gamma_{0}\left|h_{\max }\right|^{2}, \gamma_{0}=E_{b} / N_{0}$ é a razão entre a energia de bit média e a densidade espectral de potência do ruído, $Q\left(\sqrt{2 \gamma_{b}}\right)$ é a BER instantânea baseada em uma única realização de canal e $\left|h_{\max }\right|^{2}$ é a segunda estatística ordenada das normas quadráticas dos coeficientes do canal [21], i.e., ela é a variável aleatória que representa a maior de duas variáveis aleatórias qui-quadráticas com dois graus de liberdade e valor esperado unitário.

Lembrando-se que foi assumido $P_{+} \gg P_{-}$, a BER instantânea para o símbolo $s_{2}$ é dada por

$$
P_{\left(e_{2} \mid h\right)}=Q\left(\sqrt{2 \gamma_{2}}\right)
$$

em que

$$
\gamma_{2}=\gamma_{0} P\left|h_{\max }\right|^{2} .
$$

A BER média para o símbolo $s_{2}$ é então

$$
P_{e_{2}}=P_{e}\left(\gamma_{0} P\right) \text {. }
$$

em que $P_{e}(\cdot)$ foi definida em (9).

Deve-se notar que a probabilidade de se detectar erroneamente o símbolo $s_{1}$ depende de a remoção da interferência em (5) ser bem sucedida ou não. A BER instantânea para o símbolo $s_{1}$ é então dada por:

$$
\begin{aligned}
& P_{\left(e_{1} \mid h\right)}=Q\left(\sqrt{2 \gamma_{1}}\right)\left(1-P_{\left(e_{2} \mid h\right)}\right) \\
& +Q\left(\sqrt{\frac{2\left|\sqrt{P_{+} E_{b}} h_{\max }+2 \sqrt{P_{-} E_{b}} h_{\min }\right|^{2}}{N_{0}}}\right) P_{\left(e_{2} \mid h\right)},
\end{aligned}
$$

em que $\gamma_{1}=\gamma_{0} P_{+}\left|h_{\max }\right|^{2}, \quad \Delta_{i}=\sqrt{P_{+} E_{b}} h_{\max }+$ $2(-1)^{i} \sqrt{P_{-} E_{b}} h_{\min }$ e $\Pi_{h_{\max }}\left(\Delta_{i}\right)$ denota a projeção de $\Delta_{i}$ sobre o eixo real, conforme é mostrado na Figura 2. No segundo termo de (13), considera-se o fato de que, para a modulação BPSK, se a interferência não é apropriadamente removida, então a interferência remanescente torna-se duas vezes maior. $\mathrm{O}$ índice $i$ de $\Delta_{i}$ indica se os símbolos $s_{1}$ e $s_{2}$ possuem os mesmos valores $(i=0)$ ou se possuem valores opostos $(i=1)$.

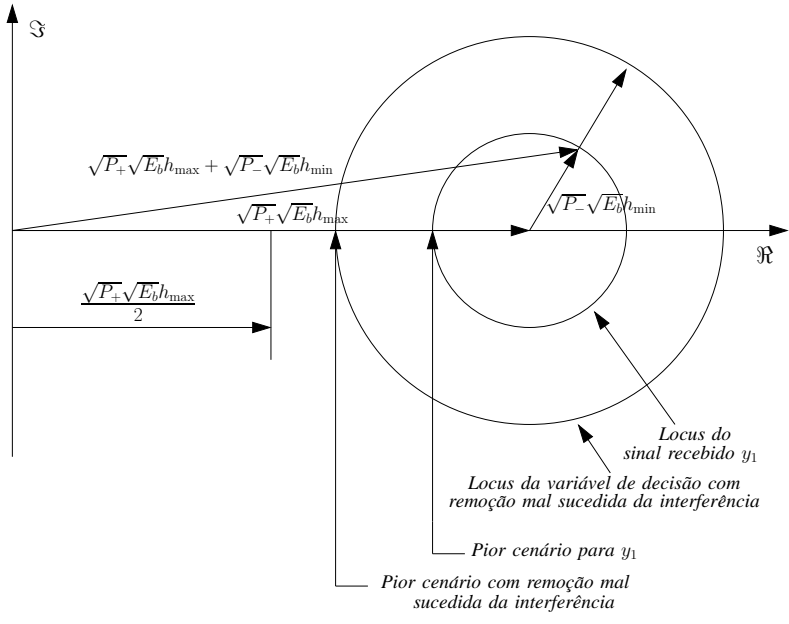

Fig. 2. $\hat{s}_{1}$ sob a condição de $P_{+} \geq 16 P_{-}$e modulação BPSK.

A seguir, uma série de desigualdades serão apresentadas com o objetivo de se encontrar um limitante superior para a BER instantânea em (13). Para ambos os casos, $i=0$ ou $i=1$, pode-se facilmente mostrar que

$$
\begin{aligned}
\mid \sqrt{P_{+} E_{b}} h_{\max }+ & \left.2 \sqrt{P_{-} E_{b}} h_{\min }\right|^{2} \\
\geq & \left(\sqrt{P_{+} E_{b}}\left|h_{\max }\right|-2 \sqrt{P_{-} E_{b}}\left|h_{\min }\right|\right)^{2}
\end{aligned}
$$

em que a igualdade ocorre se, e somente se, $h_{\max }\left|h_{\min }\right|=$ $-h_{\min }\left|h_{\max }\right|$. O lado direito da desigualdade acima corresponde ao pior cenário sob a condição de remoção mal sucedida da interferência, conforme é ilustrado na Figura 2. Se ainda for considerado que $P_{+} \geq 16 \frac{\left|h_{\min }\right|^{2}}{h_{\max }^{2}} P_{-}$(ou ainda $P_{+} \geq 16 P_{-}$) (veja a Figura 2), então, têm-se a última desigualdade das séries, a saber,

$$
\left(\sqrt{P_{+} E_{b}}\left|h_{\max }\right|-2 \sqrt{P_{-} E_{b}}\left|h_{\text {min }}\right|\right)^{2} \geq \frac{P_{+} E_{b}}{4}\left|h_{\max }\right|^{2} .
$$

Para essas desigualdades, a expressão da BER instantânea em (13) para o símbolo $s_{1}$ pode ser limitada superiormente como

$$
\begin{aligned}
& P_{\left(e_{1} \mid h\right)} \leq Q\left(\sqrt{2 \gamma_{1}}\right)\left(1-Q\left(\sqrt{2 \gamma_{2}}\right)\right) \\
&+Q\left(\sqrt{\frac{\gamma_{1}}{2}}\right) Q\left(\sqrt{2 \gamma_{2}}\right) .
\end{aligned}
$$

Um limitante superior da expressão da BER média para o símbolo $s_{1}$ pode ser obtida tomando-se a esperança de (14). Assim, obtém-se

$$
\begin{aligned}
P_{e_{1}} \leq E\{ & \left.Q\left(\sqrt{2 \gamma_{1}}\right)\right\} \\
- & E\left\{Q\left(\sqrt{2 \gamma_{1}}\right) Q\left(\sqrt{2 \gamma_{2}}\right)\right\} \\
+ & E\left\{Q\left(\sqrt{\frac{\gamma_{1}}{2}}\right) Q\left(\sqrt{2 \gamma_{2}}\right)\right\} .
\end{aligned}
$$

O resultado final é dado como uma função de $P_{s}^{(2)}\left(\gamma_{0}\right)$ em (9) e de $\Omega(\alpha, \beta)$, que é definida como

$$
\Omega(\alpha, \beta):=E\left\{Q\left(\sqrt{\alpha\left|h_{\max }\right|^{2}}\right) Q\left(\sqrt{\beta\left|h_{\max }\right|^{2}}\right)\right\},
$$




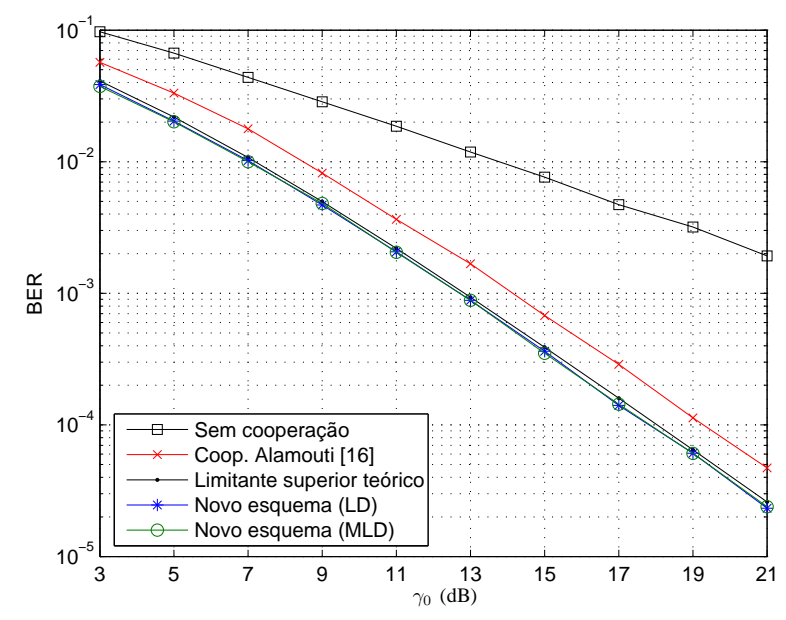

Fig. 3. BER versus SNR para o novo esquema de diversidade cooperativa e para o esquema de diversidade cooperativa com o código de Alamouti [15] (sem bit de realimentação) considerando-se um canal inter-usuário ideal. A alocação de potência adotada é de $P_{+}=0.95$.

para a qual uma expressão exata está demonstrada no Apêndice. Logo,

$$
\begin{gathered}
P_{e_{1}} \leq P_{e}\left(\gamma_{0} P_{+}\right)-\Omega\left(2 \gamma_{0} P_{+}, 2 \gamma_{0} P\right) \\
+\Omega\left(\gamma_{0} P_{+} / 2,2 \gamma_{0} P\right) .
\end{gathered}
$$

Por simetria, a expressão da BER média para uma modulação BPSK do novo esquema pode ser limitada superiormente através da média aritmética de (12) e (16).

\section{Resultados de Simulação}

Nesta seção, apresentam-se alguns resultados de simulação para se quantificar os benefícios trazidos por um único bit de realimentação em um sistema de comunicação com cooperação descrito na Seção II. Compara-se o novo esquema de diversidade cooperativa, apresentado na Seção III, com o esquema de diversidade cooperativa que utiliza o código de Alamouti [15] (sem bit de realimentação) em termos de BER versus SNR $\left(\gamma_{0}\right)$ para uma modulação BPSK sob o efeito de um desvanecimento do tipo Rayleigh plano e quaseestático. Objetivando-se visualizar o ganho de diversidade cooperativa, a curva de BER versus SNR para o esquema sem cooperação (cada nó transmite seu próprio símbolo em intervalos de símbolos distintos) é apresentado também. $\mathrm{Na}$ Figura 3, o esquema de diversidade cooperativa com o código de Alamouti [15] (sem bit de realimentação) e o novo esquema de diversidade cooperativa para $b=1$ bit de realimentação são considerados, assumindo-se que o canal inter-usuário é ideal. Resultados são apresentados tanto para o detector LD quanto para o detector MLD. O limitante superior teórico é apresentado também, dando uma idéia de sua "acurácia". A alocação de potência adotada nesta simulação é de $P_{+}=0,95$, onde a potência total é unitária $(P=1)$. Pode-se observar que o detector linear apresenta um excelente desempenho de erro. Observa-se, também, que utilizando-se apenas um único bit de realimentação o esquema proposto apresenta um ganho de
SNR sobre o código de Alamouti de aproximadamente 1,8 dB.

Vale ressaltar que no caso em que o canal inter-usuário é ideal, a alocação de potência $P_{+}$pode assumir um valor arbitrariamente alto, o qual melhoraria ainda mais o desempenho de erro do novo esquema de cooperação. Porém, para o caso em que o canal inter-usuário é real, a potência $P_{-}$ não pode ser muito baixa, pois as decisões errôneas no nó parceiro comprometeriam o desempenho total do sistema. Deve-se esperar que na presença de um canal inter-usuário real a potência $P_{+}$possa ser diminuída conforme a SNR média do canal direto decresça, ou conforme o canal inter-usuário se torne mais ruidoso. A alocação ótima de potência como uma função das estatísticas do canal inter-usuário e da SNR média no nó destino parece ser um problema muito interessante, o qual está atualmente sobre investigação.

\section{CONClusão E COMEnTÁRIOS FinAis}

Neste artigo, propôs-se um esquema simples de diversidade cooperativa para um sistema de comunicação consistindo em dois nós cooperadores que recebem um único bit de realimentação do nó destino com informação sobre o estado do canal. Esta informação é usada apropriadamente para se obter ganhos de diversidade cooperativa e de razão sinal-ruído. Um receptor linear é proposto e seu desempenho de erro mostrase ser muito próximo do desempenho obtido pelo detector de máxima verossimilhança. Um limitante superior da probabilidade de erro média para uma modulação BPSK em canais com desvanecimento Rayleigh plano, sob a condição de canais inter-usuários ideais, foi derivado. A partir dos resultados de simulação e das análises de desempenho de erro, mostrou-se que, a ordem de diversidade máxima é alcançada (igual a 2) é alcançada pelo esquema proposto. Outro importante resultado é que o novo esquema de diversidade cooperativa, fazendo uso de apenas um bit de realimentação, oferece um ganho em SNR de aproximadamente $1,8 \mathrm{~dB}$ sobre o esquema cooperativo que faz uso do código de Alamouti, ambos considerando canais inter-usuários sob condições ideais.

\section{AgRAdECIMENTOS}

Os autores agradecem ao Dr. Tolga M. Duman, da Arizona State University (ASU), AZ, Estados Unidos, por sua significativa contribuição para o desenvolvimento deste trabalho. Este trabalho foi financiado em parte pelo Conselho Nacional de Desenvolvimento Científico e Tecnológico (CNPq) sob os números de processo: 484391/2006-2 e 302286/2004-7, e pela CAPES sob o número de processo: 0176-08-7.

\section{APÊNDICE}

Neste apêndice, deriva-se uma solução exata para

$$
\Omega(\alpha, \beta)=E\{Q(\sqrt{\alpha X}) Q(\sqrt{\beta X})\},
$$

em que $X=\left|h_{\max }\right|^{2}$, definida na Seção IV, e $\alpha$ e $\beta$ são números reais positivos. A esperança é com relação a $X$, cuja PDF é dada por [10], [17], [21]:

$$
p_{X}(x)=2\left(1-e^{-x}\right) e^{-x}, \quad x \geq 0 .
$$


A função geradora de momentos de $X$, denotada por $\Psi_{X}(v)$, e pode ser definida para $v \geq 0$ conforme

$$
\Psi_{X}(v):=E\{\exp (-v X)\}=\int_{0}^{\infty} e^{-v x} p_{X}(x) d x,
$$

$$
\begin{aligned}
\Omega_{2}(\alpha, \beta)=\frac{1}{4} & -\frac{1}{2 \pi} \tan ^{-1}\left(\sqrt{\frac{\beta}{\alpha}}\right) \\
& -\frac{\sqrt{\alpha}}{\pi \sqrt{\alpha+2}} \tan ^{-1}\left(\sqrt{\frac{\alpha+2}{\beta}}\right) \\
& +\frac{1}{2 \pi} \frac{\sqrt{\alpha}}{\sqrt{\alpha+4}} \tan ^{-1}\left(\sqrt{\frac{\alpha+4}{\beta}}\right) .
\end{aligned}
$$

Agora, recorre-se a formula de Craig [18] para a função Gaussiana $Q$ :

$$
Q(x)=\frac{1}{\pi} \int_{0}^{\pi / 2} \exp \left(-\frac{x^{2}}{2 \sin ^{2} \theta}\right) d \theta .
$$

\section{REFERÊNCIAS}

[1] I. E. Telatar, "Capacity of multi-antenna Gaussian channels," Europ. Trans. Telecommun., vol. 10, pp. 585-595, Nov. 1999.

Também, foi mostrado [19, eq. (4.8)] que

$$
\begin{gathered}
Q\left(x_{1}\right) Q\left(y_{1}\right)=\frac{1}{2 \pi} \int_{0}^{\tan ^{-1} y_{1} / x_{1}} \exp \left(-\frac{y_{1}^{2}}{2 \sin ^{2} \theta}\right) d \theta \\
+\frac{1}{2 \pi} \int_{0}^{\pi / 2-\tan ^{-1} y_{1} / x_{1}} \exp \left(-\frac{x_{1}^{2}}{2 \sin ^{2} \theta}\right) d \theta
\end{gathered}
$$

Substituindo-se $x_{1}=\sqrt{\alpha X}$ e $y_{1}=\sqrt{\beta X}$ em (18), têm-se que

$$
\begin{aligned}
Q(\sqrt{\alpha X}) Q(\sqrt{\beta X}) & =\frac{1}{2 \pi} \int_{0}^{\kappa_{1}} \exp \left(-\frac{\beta X}{2 \sin ^{2} \theta}\right) d \theta \\
+ & \frac{1}{2 \pi} \int_{0}^{\kappa_{2}} \exp \left(-\frac{\alpha X}{2 \sin ^{2} \theta}\right) d \theta
\end{aligned}
$$

em que $\kappa_{1}=\tan ^{-1} \sqrt{\beta / \alpha}$ e $\kappa_{2}=\frac{\pi}{2}-\tan ^{-1} \sqrt{\beta / \alpha}$.

Tomando a esperança de (19), e usando (17), obtém-se

$$
\Omega(\alpha, \beta)=\Omega_{1}(\alpha, \beta)+\Omega_{2}(\alpha, \beta),
$$

em que

$$
\Omega_{1}(\alpha, \beta)=\frac{1}{2 \pi} \int_{0}^{\kappa_{1}} \Psi_{X}\left(\frac{\beta}{2 \sin ^{2} \theta}\right) d \theta
$$

e

$$
\Omega_{2}(\alpha, \beta)=\frac{1}{2 \pi} \int_{0}^{\kappa_{2}} \Psi_{X}\left(\frac{\alpha}{2 \sin ^{2} \theta}\right) d \theta .
$$

A partir de (17) e das integrais acima, pode-se ver que é necessário se resolver uma integral da seguinte forma

$$
\begin{aligned}
& \int \frac{2}{C+\frac{\beta}{2 \sin ^{2} \theta}} d \theta \quad C=1,2 \\
& =\frac{1}{C} \int \frac{4 C \sin ^{2} \theta+2 \beta-2 \beta}{\beta+2 C \sin ^{2} \theta} d \theta,
\end{aligned}
$$

a qual resulta em [20]:

$$
\frac{2 \theta}{C}-\frac{2 \sqrt{\beta}}{C \sqrt{2 C+\beta}} \tan ^{-1}\left(\sqrt{1+\frac{2 C}{\beta}} \tan (\theta)\right) .
$$

De (23) e (24), pode-se escrever (21) e (22) como

$$
\begin{aligned}
\Omega_{1}(\alpha, \beta)=\frac{1}{2 \pi} & \tan ^{-1}\left(\sqrt{\frac{\beta}{\alpha}}\right) \\
& -\frac{\sqrt{\beta}}{\pi \sqrt{\beta+2}} \tan ^{-1}\left(\sqrt{\frac{\beta+2}{\alpha}}\right) \\
& +\frac{\sqrt{\beta}}{2 \pi \sqrt{\beta+4}} \tan ^{-1}\left(\sqrt{\frac{\beta+4}{\alpha}}\right)
\end{aligned}
$$

[2] G. J. Foschini, and M. J. Gans, "On limits of wireless communications in a fading environment when using multiple antennas," Wireless Pers. Commun., vol. 6, no. 3, pp. 311-335, Mar. 1998.

[3] A. Sendonaris, E. Erkip, and B. Aazhang, "User cooperation diversity Part I: System description," IEEE Trans. Commun., vol. 51, no. 11, pp. 1927-1938, Nov. 2003.

[4] — "User cooperation diversity-Part II: Implementation aspects and performance analysis," IEEE Trans. Commun., vol. 51, no. 11, pp. 1939-1948, Nov. 2003.

[5] J. N. Laneman, G. W. Wornell, and D. N. C. Tse, "An efficient protocol for realizing cooperative diversity in wireless networks," in Proc. IEEE Int. Symp. Inf. Theory, 2001 (ISIT'01), p. 294, Washington, DC, Jun. 2001.

[6] J. N. Laneman, D. N. C. Tse, and G.W.Wornell, "Cooperative diversity in wireless networks: Efficient protocols and outage behavior," IEEE Trans. Inf. Theory, vol. 50, no. 12, pp. 3062-3080, Dec. 2004.

[7] V. Tarokh, H. J. Jafarkhani and A. R. Calderbank, "Space-time block codes from orthogonal designs," IEEE Trans. Inf. Theory, vol. 45, no. 5, pp. 1456-1467, July 1999.

[8] S. M. Alamouti, "A simple transmit diversity technique for wireless communications," IEEE Journal on Selected Areas on Commun., vol. 16, no. 8, pp. 1451-1458, Oct. 1998.

[9] T. E. Hunter and A. Nosratinia, "Cooperation diversity through coding," in Proc. IEEE Int. Symp. Inf. Theory, 2002 (ISIT'02), p. 220, Laussane, Switzerland, Jun./Jul. 2002

[10] D. Gore and A. Paulraj, "MIMO antenna subset selection with space-time coding," IEEE Trans. Signal Proces., vol. 50, no. 10, pp. 2580-2588, Dec. 2002.

[11] T. M. Duman and A. Ghrayeb, Coding for MIMO Communication Systems, John Wiley \& Sons, Inc., Nov. 2007.

[12] T. E. Hunter and A. Nosratinia, "Diversity through coded cooperation," IEEE Trans. Wireless Commun., vol. 5, no. 2, pp. 283-289, Feb. 2006.

[13] Y. Cao, B. Vojcic, and M. Souryal, "User-cooperative transmission with channel feedback in slow fading environment," in Proc. IEEE Vehicular Technology Conference, 2004 (VTC'04-Fall), vol. 3, pp. 2063-2067, Los Angeles, CA, Sept. 2004.

[14] N. Ahmed, M. A. Khojastepour, A. Sabharwal, and B. Aazhang "Outage minimization with limited feedback for the fading relay channel," IEEE Trans. Commun., vol. 54, no. 4, pp. 659-669, April 2006.

[15] L. Tao, L. Xiang-Ming, and Y. Guang-Xin, "Performance estimating for space-time block coded cooperative communication system" in Proc. IEEE International Conference on Communications, Circuits and Systems, 2006 (ICCCAS'06), pp. 1031-1034, Guilin, China, Jun. 2006.

[16] T. Unger and A. Klein, "Cooperative MIMO Relaying with distributed space-time block codes" in Proc. The 17th Annual IEEE International Symposium on Personal, Indoor and Mobile Radio Communications, 2006 (PIMRC'06), pp. 1-5, Helsinki, Finland, Sept. 2006.

[17] Z. Chen, J. Yuan, and B. Vucetic, "Analysis of transmit antenna selection/maximal-ratio combining in Rayleigh fading channels ," IEEE Trans. Vehic. Technology, vol. 54, pp. 1312-1321, July 2005.

[18] J. W. Craig, "A new, simple and exact result for calculating the probability of error for two-dimensional signal constellations," IEEE Military Commun. Conf., 1991 (MILCOM'91) Conf. Rec., pp. 571-575, Boston, MA, Nov. 1991.

[19] M. K. Simon and M.-S. Alouini, Digital Communications over Fading Channels, John Wiley \& Sons, Inc., New York, 2000.

[20] M. R. Spiegel and J. Liu, Mathematical Handbook of Formulas and Tables, 2nd edition, McGraw-Hill, 1999.

[21] H. A. David and H. N. Nagaraja, Order Statistics, Third Edition, Wiley Series in Probability and Statistics, John Wiley \& Sons, Inc., 2003. 